\title{
El turismo en las dos orillas del Mediterráneo (Andalucía-Norte de Marruecos): Cooperación Transfronteriza entre el Instituto Superior de Turismo de Tánger y la Universidad de Almería
}

\author{
González-Alarcón, Isabel Esther
}

Universidad de Almería, igonzale@ual.es

\begin{abstract}
Resumen
El Instituto Internacional de Turismo de Tánger (ISITT) y la Universidad de Almería (UAL) poseen en común intereses y finalidades de formación académicas científicas y culturales. Es por ello que se llevará a cabo el intercambio de docentes e investigadores entre las dos entidades durante un espacio de tiempo determinado. Del mismo modo, cada una de las dos instituciones facilitará la publicación conjunta de libros y trabajos respetando las normas en vigor. El trabajo que presentamos aquí forma parte del Proyecto de Cooperación Transfronteriza PARALELO 36 (CIME-AM) cuyo principal beneficiario es la Universidad de Almería (UAL) a través del CEMyRI (Centro de Estudios Migratorios y de Relaciones Interculturales) de la Universidad de Almería. El objetivo principal de este artículo es el de mostrar los resultados de la Actividad 2 del proyecto: «Elaboración conjunta de herramientas duraderas sobre el turismo y el trabajo social para poner en común criterios relacionados con la competencia técnica en el dominio de la transnacionalidad, en el Mediterráneo occidental», dos disciplinas de enorme arraigo entre la población y las instituciones en ambas orillas del Mediterráneo.
\end{abstract}

Palabras clave: Mediterráneo; turismo; ISITT; UAL; transnacionalidad.

\section{Résumé}

L'Institut International du Tourisme de Tanger (ISITT) et l'Université de Almería (UAL) ont en commun des intérêts et des finalités de formation académiques scientifiques et culturels. C'est ainsi que l'on favorisera l'échange d'enseignants et de chercheurs entre les deux Institutions, de façon à faciliter l'intervention de professeurs entre les deux établissements pour une durée déterminée. De la même façon, chacune des deux Institutions facilitera la publication conjointe de livres et de travaux dans le respect des normes en vigueur. Le travail que nous présentons ici fait partie du Projet de Coopération Trasnfrontalière PARALELO 36 (CIME-AM), dont le principal bénéficiaire est l'Université de Almería (UAL) à travers le CEMyRI (Le Centre d'Études des Migrations et des Relations Interculturelles) de l'Université de Almería. L'objectif principal de cet article est celui de montrer les résultats de l'Activité 2 de ce projet: «Production en commun d'outils durables centrés sur le tourisme et le travail social pour mettre en commun des critères en ce qui concerne la compétence technique dans le domaine de la transnationalité, avec un intérêt spécial pour la Méditerranée occidentale», deux disciplines profondément enracinées dans la population et les institutions des deux rives de la Méditerranée.

Mots-clés: Méditerrané; tourisme; ISITT; UAL; transnationalité.

\begin{abstract}
The International Institute of Tourism of Tanger (ISTT) and the University of Almeria (UAL) share interests and scientific, cultural and academic objectives. For this purpose, international exchanges between professors and researchers from the two entities will take place during a period of time. Similarly, both institutions will facilitate co-publications of books and projects according to the established norms. The present paper forms part of the Cross-border Cooperation Project PARALELO 36 (CIME-AM) holding the University of Almeria as principal beneficiary through CEMyRI (Migration Studies and Intercultural Relations Centre). The main objective of this article is to provide results about Activity 2 of the project:
\end{abstract}


El turismo en las dos orillas del Mediterráneo (Andalucía-Norte de Marruecos). Cooperación transfronteriza entre el Instituto Superior de Turismo de Tánger y la Universidad de Almería.

«Joint design of long-term tools for tourism and social work to share criteria related to the technical competence in the domain of transnationality in the West Mediterranean». These two disciplines are strongly rooted in the population and institutions of both Mediterranean sides.

Keywords: Mediterranean; tourism; ISITT; UAL; transnationality.

\title{
Introducción
}

\begin{abstract}
África, 14 km al sur de Europa...es el título de un trabajo de investigación y es también la misma distancia que media entre el punto más meridional de la costa andaluza y el más septentrional del litoral marroquí. Y lo que en el primer caso se traduce a través del compás de las alegrías, si el segundo llevase banda sonora probablemente sonaría a seguiriya o a una de esas salmodias de las nubas andalusíes que conservan heroicas orquestas populares de Tetuán o de Tánger, alabada sea la memoria de los maestros Chekara y Tensamani. Ese ritmo sucesivo vendría bien para acunar la larga secuencia de sueños, de aventuras personales y también de muerte que entraña el cruce de la frontera sur de Europa (González y Vera, 2008: 11).
\end{abstract}

El viaje del Mediterráneo es, por fuerza, un recorrido literario. No puede uno navegar sus aguas ni recorrer sus litorales sin escuchar el alma de literatura. Muchos de los grandes caminos del mar de la literatura salen desde sus puertos o van a morir en sus orillas.

También es esperanza o corredor de la muerte para, cada vez, un mayor número de inmigrantes que, bien huyendo de países en conflicto o de la pobreza arriesgan su vida cruzando el mar.

Como bien citaba el poeta Francisco Checa desde un atardecer tangerino que compartimos, sobre las tumbas del Hafa, al otro lado del Estrecho. Nunca una corta lejanía / fue tan inalcanzable / Nadando atravieso tu garganta / de un salto si quisiera / Las mujeres
y ancianos también pasan / Y se sientan sobre siglos, / y alegres, junto a sus hijos / devoran pipas y, a veces, se ríen /
¡Qué Estrecho tan inalcanzable! / Ayer, que fue hermano nuestro, / y guía, y asidero, y sempiterno aliado / Hoy, un
cementerio / Y el teléfono suena / y alguien habla / y saluda al infinito / con el brazo extendido, /señal de amor, a lo
lejos / En dos brazadas / si quisiera... (Checa, 2015: 98).

De la unión de este Estrecho, nosotros nos centraremos en dos puntos geográficos, Marruecos y el sur de España, Andalucía: y más concretamente en las ciudades de Tánger y Almería.

Tomando como eje central las aguas del Mediterráneo y las dos orillas, en este artículo se expondrán los resultados obtenidos de la elaboración de materiales conjunta sobre los estudios de Turismo que el ISITT y la Universidad de Almería han llevado a cabo desde el 1 de enero de 2012 hasta el 31 de diciembre de 2013, dentro del Proyecto de Investigación (cuyo acrónimo es PARALELO 36 CIME-AM), y en donde la autora de esta comunicación ha sido investigadora colaboradora así como coordinadora interuniversitaria en la elaboración de tales materiales docentes.

Del mismo modo hablaremos del intercambio de estudiantes marroquíes y andaluces de la especialidad de Turismo en su período de prácticas que han venido realizando tanto en empresas turísticas tangerinas como almerienses durante el periodo de colaboración. También haremos mención a las actividades docentes e investigadoras conjuntas que tanto estudiantes y profesores-investigadores del Instituto Superior de Turismo de Tánger como del Grado de Turismo de la Universidad de Almería realizaron.

El Proyecto Capacitación y estrategias para el trabajo en intervención social y preparación para la mejora del empleo en las dos orillas (Andalucía-Norte de Marruecos), se enmarca dentro del Programa Operativo de Cooperación Transfronteriza España - Fronteras exteriores (POCTEFEX). Éste estuvo financiado por el Fondo Europeo de Desarrollo Regional (FEDER), a través del programa de Cooperación Transfronteriza España-Fronteras Exteriores (POCTEFEX). 
Dicho proyecto, liderado por la Universidad de Almería, a través del Centro de Estudios de las Migraciones y las Relaciones Interculturales (CEMyRI), contó como socios andaluces con: la Dirección General de Coordinación de Políticas Migratorias (Consejería de Justicia e Interior de la Junta de Andalucía), la Agencia Pública Empresarial Sanitaria Hospital de Poniente (Consejería de Salud de la Junta de Andalucía) y como socios marroquíes con El Instituto Nacional de Acción Social de Tánger (INAS) y el Instituto Superior de Estudios de Turismo de Tánger (ISITT).

Entre sus objetivos principales, se hallaba el hecho de estimular, mejorar y coordinar las estrategias de intervención social y sanitaria (con mujeres y menores-jóvenes en Marruecos e inmigrados marroquíes en Andalucía) y mejorar la formación y el acceso al empleo de la población en ambas orillas en torno al turismo.

Nosotros, de este gran conjunto intercultural nos centraremos en la Actividad 2 del proyecto y dentro de su Actividad 2, en la unión e intercambio de las tareas docentes, profesionales y de investigación llevadas a cabo entre el ISITT y la Universidad de Almería.

\section{Intercambio de estudiantes universitarios}

Dentro de la Actividad 2, la Acción 1 consistía en la realización de prácticas profesionales de estudiantes de turismo y trabajo social andaluces y marroquíes, concretamente doscientas horas por estudiante, durante un mes.

De este modo, en 2012, un máximo de treinta estudiantes universitarios (quince de Almería y quince de Tánger) realizaron prácticas interprofesionales en entidades del sector turístico y la intervención social en el país vecino.

Igualmente, en 2013, se desarrolló la segunda parte del Intercambio de estudiantes, donde treinta alumnos (quince de Almería, y quince de Tánger) de las titulaciones de Turismo y Trabajo Social culminaron sus prácticas afines a su titulación en el país vecino.

Cito textualmente un fragmento de la noticia Un grupo de estudiantes marroquíes realiza desde hoy sus prácticas profesionales en la UAL:

El CEMyRI oferta 30 estancias de prácticas profesionales para estudiantes universitarios en un intercambio entre Almería y el Norte de Marruecos.

Aunque la distancia entre Almería y el Norte de Marruecos es corta, la Universidad de Almería a través del Centro de Estudios de la Migraciones y las Relaciones Interculturales (CEMyRI) acercará aún más las dos orillas a través de un intercambio de estudiantes universitarios.

El Centro de estudios de las Migraciones y Relaciones Interculturales ha ofertado treinta estancias para la realización de prácticas profesionales que están enmarcadas en el proyecto Capacitación y Estrategias para el trabajo en intervención social y preparación para la mejora del empleo, PARALELO 36 CIME-AM.

Los alumnos de la Universidad de Almería, quince en total, y pertenecientes a los estudios de Grado en Trabajo Social, Turismo, Máster en Estudios Migratorios y Doctorado en Estudios Migratorios realizarán un máximo de 200 horas de prácticas durante un mes en Marruecos. Y los alumnos, otros quince, del Instituto Nacional de Acción Social y del Instituto Superior de Estudios de Turismo, entidades marroquíes asociadas también al proyecto, optarán al mismo periodo de prácticas en Almería durante el mes de septiembre.

El proceso de selección de los solicitantes ha sido llevado a cabo tanto por la Universidad de Almería como por el Instituto Nacional de Acción Social y el Instituto Superior de Estudios de Turismo. Entre los requisitos para optar a una vacante se encontraban la nota media ponderada del expediente académico, la experiencia investigadora de los alumnos y el conocimiento de idiomas de éstos, ante todo árabe y/o francés. Además de la realización de un proyecto individual a cerca de su perfil profesional en relación a su posible estancia práctica. (http://cms.ual.es/UAL/universidad/organosgobierno/gabcomunicacion/noticias/18SEP2012_MARROQUIES). 
El turismo en las dos orillas del Mediterráneo (Andalucía-Norte de Marruecos). Cooperación transfronteriza entre el Instituto Superior de Turismo de Tánger y la Universidad de Almería.

El periódico digital Almería 360 especificaba:

La Universidad de Almería, a través del CEMyRI, Centro de Estudios de las Migraciones y las Relaciones Interculturales, ha dado la bienvenida este lunes, día 13 de Mayo a ocho estudiantes marroquíes del Instituto Nacional de Acción Social (INAS) de la ciudad de Tánger, que tendrán la oportunidad de realizar sus prácticas profesionales en distintas entidades de la provincia de Almería relacionadas con la intervención social y la mediación. El 7 de Junio llegarán el segundo grupo de estudiantes, alumnos de Instituto Superior de Estudios de Turismo (ISITT), que pasarán otro mes en nuestra provincia completando su formación profesional.

(...) Los estudiantes se incorporarán el martes 14 a sus lugares de prácticas: Asociación Almería Acoge, Asociación de Personas con Discapacidad “El Saliente”, Agencia Pública Empresarial Sanitaria Hospital de Poniente, Federación Almeriense de Asociaciones de Personas con Discapacidad y la Asociación para la Lucha de Enfermedades de Riñón (Alcer).

Por otra parte, los alumnos del Instituto Superior de Estudios de Turismo de Tánger (ISITT), ya pudieron realizar sus prácticas en Almería. Durante su breve pero intenso periplo, los jóvenes tangerinos se integraron con la población local a través de los roles que han desempeñado en los puestos de trabajo que se le asignaron. Los estudiantes pudieron realizar sus prácticas en diferentes empresas de la provincia tales como Indalcongress, Viajes Alcazaba, Hotel Bellavista Roquetas de Mar, Fundación Bahía Almeriport y Hotel Costasol.

El CEMyRI ha gestionado esta estancia y ha contado con la colaboración de la Fundación Mediterránea Empresa Universidad de Almería. La duración de las prácticas será de un mes, hasta el 7 de mayo, fecha en la que partirán los alumnos a su ciudad de origen para incorporarse a sus respectivos estudios. Asimismo, a finales de Septiembre, un total de 15 estudiantes de la Universidad de Almería, realizarán su estancia en Marruecos, todo ello dentro del mismo programa de Cooperación. (http://almeria360.com/educacion/25072012 almeria-y-marruecos-intercambianestudiantes-en-periodo-de-practicas 32444.html).

De los resultados de esta Acción 1 del proyecto hemos de decir que la posibilidad de realizar prácticas en un país extranjero ha sido una gran oportunidad para nuestros alumnos del Grado de Turismo de la Universidad de Almería y no sólo por adquirir experiencia profesional, tan solicitada actualmente, sino también por conocer e interiorizar otras culturas y estilos de vida, algo fundamental para todos aquellos que enfocan su carrera profesional hacia el sector turístico y de ayuda social.

En cada una de las actividades descritas en este convenio, las distintas entidades designaron a un coordinador que se responsabilizó del seguimiento de las actividades.

Todas las partes se comprometieron a acreditar el trabajo por el alumnado, en los términos en que dicha participación se produjo.

Los coordinadores de prácticas designados exigieron a los alumnos cumplir con el horario establecido, cumplir con el orden y disciplina interna de cada Institución y cumplir las directrices marcadas por el director de prácticas en relación con las líneas de trabajo pactadas.

Los estudiantes, del mismo modo, estuvieron obligados a cumplir con el horario pactado con las distintas entidades para la realización de prácticas, con la norma de disciplina interna, con las tareas inherentes a las líneas de trabajo para las que habían sido seleccionados, con las normas de seguridad e higiene en el trabajo y con el desarrollo eficaz del Plan formativo previsto.

Hubo dos reuniones de seguimiento: en 2012, en Tánger (Marruecos) y en 2013, en Almería (Andalucía), respectivamente, de profesores de turismo y trabajo social o Máster para evaluar la experiencia del estudiantado.

Cito, al respecto, un fragmento del Acta de la Reunión mantenida en Tánger en la sede del ISITT y en relación al primer punto del Orden de día (Évaluation du Stage des élèves marocains à Almería):

Mme. Canton, coordinatrice du Stage des élèves marocains à Almería, a félicité les enseignants marocains pour le bon comportement de leurs élèves, aussi bien dans leurs lieux l'hébergement que dans les entreprises de Almería où ils ont réalisé leur stage. « Ils sont rentrés très contents » a ajouté Mme. AMAL. Comme il était prévu, indique Mme Cinep BECERRADA, ces élèves rédigent actuellement un rapport dans lequel ils exposent leur expérience de stage à Almería, au niveau social et au niveau du travail. Mme. AMAL affirme qu'il est très important que les élèves survivent dans un 
autre environnement et qu'ils mettent en perspective ce qui se passe dans les entreprises espagnoles par rapport aux marocaines.

Pour conclure, selon l'opinion des enseignants espagnols et marocains, l'évaluation de ce stage des élèves marocains à Almería a été très positive (COMPTE RENDU 9 octobre 2012. 10 heures, à l’ISITT).

Para el buen desarrollo del proyecto se acordó exigir a los estudiantes que tuvieran unos conocimientos básicos de francés, en el caso de los alumnos de la Universidad de Almería y de español, en el caso de los estudiantes tangerinos.

Del mismo modo, profesores, alumnos y profesionales de las distintas entidades se comprometieron a elaborar materiales docentes destinados a la unificación de criterios en la capacitación técnica en transnacionalidad con especial atención en el Mediterráneo Occidental. Asimismo, participaron en la elaboración, difusión y mantenimiento de las herramientas virtuales que se elaboraron en el marco del proyecto que amparaba el convenio de las citadas entidades en su Actividad 2, concretamente en la Acción 2 y que a continuación detallamos.

\section{Elaboración conjunta de herramientas duraderas sobre el Turismo y el Trabajo Social en el Mediterráneo Occidental}

En cuanto a la Elaboración de materiales docentes, se elaboró un manual con distintas temáticas de interés para las dos partes (marroquí y española), también se designaron responsables de cada línea de trabajo.

Estos materiales serían de dos tipos, una página web, propia del proyecto con acceso libre para los estudiantes y los profesores de los dos países, más un libro (Checa y Olmos, 2014) que vio la luz tras la finalización del proyecto.

Para la elaboración del libro/guía se establecieron unas directrices o capítulos guía que girarían en torno a las siguientes temáticas:

\subsection{Introducción general}

\subsubsection{Análisis socioeconómico, cultural y turístico entre España y Marruecos.}

\subsubsection{La formación en Turismo en España y Marruecos}

- $\quad$ Las escuelas de Turismo en España y Marruecos

\subsection{Legislación en materia de turismo en las dos orillas}

Dado el carácter trasversal del turismo su regulación se realiza a través de normas que forman parte de diferentes campos jurídicos. Toda persona física o jurídica que pretenda desarrollar una actividad económica basada en la prestación de servicios turísticos debe someterse, por un lado, al Derecho Privado, es decir, aquella parte del derecho que se ocupa de las relaciones entre particulares, como es la contratación turística (alojamientos, transportes, seguros...) o la responsabilidad civil de las partes por el incumplimiento de sus obligaciones. Y por otro lado, al Derecho Público, en tanto es la Administración la que actúa sobre el turismo y sobre los sujetos que operan en éste ejerciendo su autoridad a través del control que ejerce por medio de distintos cauces. Ese control es una consecuencia de los efectos que produce la actividad turística sobre los usuarios turísticos en particular y sobre la sociedad en general.

En este sentido, se llevó a cabo una descripción del tema en España y Marruecos, dado que lo que se pretendía era mostrar una visión de la legislación en materia de turismo en los dos países.

\subsection{Estructura sectorial del turismo en España y Marruecos}

En esta sección se describieron las principales empresas participantes en cada uno de los eslabones del sector turístico. En este sentido, aunque España cuenta con más de 200 cadenas hoteleras (Sol, Meliá, NH, HUSA...), sólo se plasmaron 
El turismo en las dos orillas del Mediterráneo (Andalucía-Norte de Marruecos). Cooperación transfronteriza entre el Instituto Superior de Turismo de Tánger y la Universidad de Almería.

aquellas que por volumen de negocio e internacionalización, tenían más relevancia, así como las empresas internacionales con presencia en España, analizándose la relación de estas corporaciones con el destino Marruecos.

\title{
2.4. El patrimonio cultural histórico y artístico de la zona de Almería y Tánger
}

El capítulo V del libro Patrimonio Cultural. Caso de Andalucía y el capítulo X La ville patrimoniale et tourisme. Cas de Chaouen muestran un claro ejemplo del patrimonio cultural histórico y artístico en ambas orillas, dado que:

\begin{abstract}
Cualquiera que haya sido turista o viajero alguna vez habrá comprobado que el discurso del patrimonio cultural - el folclore, las fiestas, la gastronomía o la música tradicionales- ha sido un aliado muy importante del turismo. Gracias al turismo muchos pueblos y zonas enteras han sido capaces, al mismo tiempo que mantienen sus tradiciones, de progresar social y económicamente, precisamente por mantenerlas (Checa y Arjona, 2014: 43).
\end{abstract}

Como ejemplo de ello se centraron en dos puntos geográficos: el caso de Andalucía y la ciudad de Chaouen.

\subsection{Marketing y protocolo. Comportamientos del consumidor y turista}

Los contenidos desarrollados en este tema permitieron a los alumnos conocer qué lugar ocupaba el marketing dentro de la planificación estratégica de la empresa turística y de qué manera se desarrollaba la misma desde el punto de vista de la comercialización. En el mismo, se profundizó en la comprensión de la importancia y utilidad que posee el marketing para la gestión turística, analizándose diversos elementos e instrumentos del subsistema comercial que condicionaban el desarrollo de la actividad empresarial turística, tales como el análisis del mercado y del entorno, el estudio del comportamiento del consumidor y la organización turística, la segmentación de mercados, la calidad de servicio, la gestión de las relaciones con el cliente y la investigación comercial.

Paralelamente, con este tema se quiso despertar la inquietud del alumno por conceptos empresariales tales como la diferenciación, la innovación, la competitividad y la creatividad.

\subsection{Francés para Turismo}

Este apartado era de marcado carácter obligatorio, dado que ponía de manifiesto la lengua de comunicación reinante entre ambas instituciones y entidades, de hecho todos los documentos (convenios, actas, capítulos de libro, etc.) fueron traducidos del francés al español y viceversa.

Este epígrafe se centró principalmente en el francés para fines específicos y muy especialmente en el FLE en los estudios universitarios de Turismo, en las necesidades comunicativas y en los objetivos existentes en el ámbito de la comunicación turística.

\subsection{Trabajo social del Turismo}

El nacimiento del turismo social en España tal y como lo conocemos en la actualidad está inexorablemente ligado al Trabajo Social. Fueron las trabajadoras sociales María Patrocinio Las Heras Pinilla (Directora General de Acción Social del Ministerio de Trabajo 1983-1990) y Elvira Cortajarena Iturrioz las que implantaron e impulsaron en los años 80 del siglo pasado el programa de turismo social en nuestro país.

En un principio, y para acotar el ámbito de esta línea, la propuesta se centró en el colectivo de personas mayores.

La provincia de Almería es receptora de beneficiarios del programa que contribuyen al mantenimiento de puestos de trabajo en el sector turístico, y por añadidura a la creación y consolidación de empleos indirectos. 
En relación al proyecto, la oportunidad viene determinada por la amplia experiencia de buenas prácticas en España que puede ser extrapolada a la realidad marroquí, de hecho en 2011 se celebraron los 25 años en España del programa de Turismo Social.

Existe un gran interés en el país vecino por el tema, como consecuencia de ello, el 28 de agosto de 2013 se celebró en Essaouira el Congreso Mundial de Turismo Social.

Por otro lado, también se está llevando a cabo una ampliación de oferta y circuitos, dada la proximidad geográfica con Marruecos.

Un tema bastante relevante en el momento actual dentro del sector turístico y que el alumnado de ambas orillas debe conocer en profundidad.

Finalmente, como resultado de toda esta confluencia de líneas y temáticas de investigación, entre docentes e investigadores marroquíes y españoles, ambas instituciones acordoron denominar el libro, El Turismo en el Mediterráneo Occidental - Le Tourisme à L'Ouest de la Méditerranée. Como coordinadores del mismo encontramos a docentes de las dos entidades, las profesoras Cantón Rodríguez, González Alarcón y Jover Silvestre de la Universidad de Almería y los docentes Mouhtaj y Alami del ISITT. Como coordinadora técnica, Yolanda Cara Fernández y finalmente como Director de la publicación, el profesor de la Universidad de Almería y Director del CEMyRI, Francisco Checa y Olmos.

Del mismo modo, se realizaron diversos encuentros que docentes e investigadores de las especialidades de Turismo y Trabajo Social de las dos orillas, celebraron en la Universidad de Almería, y en los cuales pusieron en común las experiencias y resultados obtenidos en él durante los dos años del proyecto.

\section{Jornadas y Congresos}

- Colaboración en la organización del XIV Congreso de Inmigración de la Universidad de Almería (2012), en donde se sufragaron tres ponencias sobre metodologías y buenas prácticas y una mesa redonda de intercambio de experiencias en el trabajo social con mujeres y menores-jóvenes en el Norte de marruecos e inmigrados marroquíes en Andalucía. Dicho Congreso, contó con una nutrida presencia de ponentes procedentes de Marruecos.

- Encuentro en Almería sobre patrimonio cultural y etnográfico y arte de las dos orillas (2012): Se llevaron a cabo diversas exposiciones de fotografía, pintura y escultura; muestra de cine andaluz y marroquí, ciclo de cine e inmigración, muestra de teatro de las dos orillas y recital de poesía andaluza-marroquí.

- Jornadas de intercambio profesional para la reflexión teórica y práctica y la evaluación del estado actual de los estudios de trabajo social en Andalucía y norte de Marruecos ( 2013) y formación en mediación transnacional: Estas jornadas sirvieron de plataforma de difusión de las nuevas herramientas enunciadas en la Acción 2 del proyecto.

Con el objetivo de que los resultados y el éxito de este proyecto europeo perdurase en nuestras clases del Grado de Turismo y no quedase en el olvido, decidí llevar a cabo con los estudiantes de tercero del Grado de Turismo en la asignatura Ampliación de Idioma moderno aplicado al sector turístico : francés un Trabajo Fin de Asignatura en donde deben hacer una introducción sobre los países africanos de habla francesa a fin de conocer sus costumbres, su cultura y sus normas sociales protocolarias, así como un proyecto fin de materia que guarde relación, evidentemente, con el sector turístico, y cuyos resultados deben exponer en francés tal cual se viene haciendo en las exposiciones de Trabajo Fin de Grado de la Universidad de Almería. Actualmente estamos en proceso de creación de un blog (http://tourismeual.gmail.com) en donde incluiremos toda esta información (las instrucciones a seguir en la búsqueda bibliográfica, cómo deben citar dentro del corpus del trabajo escrito, etc.). Para ello, nuestro alumnado deberá conocer la cultura francófona de todos los pueblos de habla francesa, centrándonos principalmente en los que se encuentran a la otra orilla del Mediterráneo, ya sean los geográficamente situados dentro de los límites del África blanca o al sur del Sáhara. La elección del país la escoge el estudiante, porque, como futuros agentes del sector turístico, deben conocer todas las costumbres del viajero francófono que viene, sea éste de nacionalidad nigeriana, o senegalesa, por ejemplo, dado que...si no conociesen sus costumbres y maneras de vivir, ¿sabrían recibirlos protocolariamente como debieran? 


\title{
Conclusión
}

Concluimos nuestro trabajo con una cita de las profesoras Cantón Rodríguez, González Alarcón y Jover Silvestre, en relación a la comunicación intercultural y al estudio de las lenguas extranjeras:

\begin{abstract}
La communication interculturelle semble répondre aux nouveaux besoins de communication. Mais le premier obstacle pour la réalisation de ces échanges est la méconnaissance de la langue de « l'autre ». Cela a provoqué le développement des langues étrangères conçues comme le moyen principal pour accéder à la culture. L'étude de langues étrangères doit être accompagnée du contexte de production. Cette condition méthodologique requise pour la communication dans les domaines professionnels du tourisme a une grande importance. Il n'y a pas les mêmes usages de la langue française dans les cas de parlants français, arabes ou canadiens, par exemple. C'est pour cette raison que la culture est complètement liée à l’apprentissage de la langue (Cantón, González y Jover, 2014: 187).
\end{abstract}

Es obvio que el estudio y conocimiento de una lengua extranjera es condición sine qua non para acceder a la cultura del Otro, de ahí que en nuestras clases de francés en el Grado de Turismo trabajemos, paralelamente, con nuestro alumnado, las costumbres y maneras de vivir de todos los pueblos francófonos.

\section{Referencias bibliográficas}

CANTÓN RodríGuez, Ma Loreto; GonZÁLEZ Alarcón, Isabel Esther y Jover SiLvestre, Yolanda (2014). «Les langues dans les Etudes Universitaires de Tourisme», en Checa y Olmos, Francisco et al. El Turismo en el Mediterráneo Occidental - Le tourisme à l'Ouest de la Méditerranée. Almería: CEMyRI.

Checa y Olmos, Francisco (2015). El mar que no piso. Almería: Círculo Rojo.

Checa y Olmos, Francisco et al. (2014). El Turismo en el Mediterráneo Occidental - Le tourisme à l'Ouest de la Méditerranée. Almería: CEMyRI.

CheCA y Olmos, Francisco y ARJona Garrido, Ángeles (2014). «Patrimonio cultural. El caso de Andalucía», en Checa y Olmos, Francisco et al. El Turismo en el Mediterráneo Occidental - Le tourisme à l'Ouest de la Méditerranée. Almería: CEMyRI.

COMPTE RENDU de 9 de octubre de 2012. 10.00h. Sede del ISITT [Consulta: 10 de abril de 2016].

GABINETE DE COMUNICACIÓN. UNIVERSIDAD DE ALMERÍA. < http://cms.ual.es/UAL/universidad/organosgobierno/gabcomunicacion/noticias/18SEP2012_MARROQUIES> [Consulta: 14 de mayo de 2016].

GonzÁlez Ferrera, Gema y Vera Borja, José Manuel (2008). África, 14 kilómetros al sur de Europa. Análisis de los flujos migratorios entre el Norte de África y el Sur de Andalucía. Sevilla: Junta de Andalucía, Consejería de Gobernación.

PERIÓDICO DIGITAL Almería $360<$ http://almeria360.com/educacion/25072012_almeria-y-marruecos-intercambian-estudiantes-enperiodo-de-practicas_32444.html> [Consulta: 14 de mayo de 2016]. 